\title{
Intensity Non-uniformity Correction for Image Segmentation
}

\author{
Jaya Krishna Sunkara ${ }^{1}$, Sundeep Eswarawaka ${ }^{2}$, Kiranmai Darisi ${ }^{2}$, Santhi Dara ${ }^{2}$, \\ Pushpa Kumar Dasari ${ }^{2}$, Prudhviraj Dara ${ }^{2}$ \\ ${ }^{I}$ Asst. Prof., Dept. of ECE, PDCE, Sullurpet, INDIA. \\ ${ }^{2}$ Student of B.Tech, ECE, PDCE, Sullurpet, INDIA.
}

\begin{abstract}
Intensity non-uniformity or intensity inhomogeneity usually occurs in Real world Images, those images cannot be segmented by using image segmentation. The most commonly used algorithms in image segmentation are region based and depends on the homogeneity of the image intensities which usually fails to produce accurate segmentation results due to the intensity non-uniformity. In this paper we proposed a novel region based method for image segmentation which can be able to discuss with intensity non-uniformities in image segmentation. First according to the image models with intensity non-uniformities we define a local clustering criterion function for the intensities in the image neighbourhood of each part. The local clustering criterion function is then integrated with respect to the neighbourhood center to give a global criterion of image segmentation. In a level set formulation this criterion defines an energy in terms of level set functions that represents the partition of image domain and a bias field that corresponds to the intensity non-uniformity of the image. Therefore, by minimizing the energy we can able to segment the image simultaneously and estimate the bias field can be used for the intensity non-uniformity correction. This method is applied on MRI images and real world images of various modalities with desirable performance in the presence of intensity nonuniformities. The experiment results show that the method is stronger, faster and more accurate than the wellknown piecewise smooth model and gives promising results. As an application this method is used for segmentation and bias correction of real world images and MRI images with better results.
\end{abstract}

Keywords -Bias field, Energy minimization, Image segmentation, Intensity non-uniformity, Level set method.

\section{Introduction}

Intensity non-uniformity frequently occurs in real world images due to the various factors such as imperfections of the imaging devices which correspond to many problems in image processing and computer vision [1]. Image segmentation may be mainly difficult for the images with intensity non-uniformities due to the overlap between the ranges of the intensity in the regions to be segmented. This makes it impossible to categorize these regions based on the pixel intensity. Those extensively used image segmentation algorithms depends on intensity homogeneity and hence not applicable to images with intensity non-uniformities .In general, intensity non-uniformity has been an interesting problem in image segmentation.

The level set method is used as a statistical technique for tracking the interfaces and shapes that has been progressively applied to image segmentation in the past years. In the level set methods surfaces are represented to the zero level set of higher dimensional function called as level set function. With level set illustration the image segmentation problem can be formulated and solved by using mathematical theories and including the partial differential equations.

The advantage of level set method is that numerical computations involves curves and surfaces which can be performed on fixed Cartesian grid without having no constraints. Existing level set methods for image segmentation can be resolute into two classes; they are Region-based models and Edge-based models. Regionbased models are used to categorize each region by using a certain region descriptors to guide the motion of the active contours. It is very difficult to define a region descriptor for images with non-uniformities. Most of the region based models are based on the assumption of intensity homogeneity. A typical example is piecewise constant models and level set methods are proposed based on general piecewise smooth formulation proposed by Mumford and shah. These methods are able to segment the images with intensity non-uniformities however these methods are worked out to be too expensive and are quite sensitive to the initialization of the contour. Edge-based models use edge information for image segmentation; those models do not assume homogeneity of image intensities and thus can be applied to images with intensity non-uniformities.

These methods are quite sensitive to initial conditions and frequently suffer from serious boundary leakage problems in images with weak object boundaries. A novel region based method for image segmentation is proposed. A local intensity clustering property and local intensity clustering criterion function for the intensities in a neighbourhood of each point is defined in this paper. This local clustering criterion is integrated over the neighbourhood center to define energy functional, which is converted to a level set formulation 
minimization of this energy is achieved by an interleaved process of level set evolution and estimation of bias field. This method is applicable to the segmentation and bias correction of MR images.

\section{RELATED WORK}

Let $\Psi$ be the image domain, and $I: \Psi \rightarrow \mathrm{R}$ be a grey level image. The segmentation of the image $I$ is achieved by finding a contour $\mathrm{C}$, which separates the image domain $\Psi$ into disjoint regions $\Psi_{1}, \Psi_{2}, \ldots \ldots \ldots ., \Psi_{\mathrm{N}}$, and a piecewise smooth function $u$ that approximates the image $I$ and is smooth inside each region $\Psi_{\mathrm{i}}$. This can be formulated as a problem of minimizing the following Mumford -Shah functional

$$
f^{M S}(u, C)=\int_{\Psi}(I-u)^{2} d x+\mu \int_{\Psi \backslash C}|\nabla u|^{2} d x+v|c|
$$

Where first term is the data term, which forces $u$ to be close to the image $I$, second term is the smoothening term, which forces $u$ to be smooth within each of the regions separated by the contour $\mathrm{C}$. The third term includes regularizing the contour $\mathrm{C},|\mathrm{C}|$ is the length of the contour $\mathrm{C}$.

Let $\Psi_{1}, \Psi_{2} \ldots \Psi_{\mathrm{N}}$, be the regions in $\Psi$ separated by the contour $\mathrm{C}$, i.e. $\Psi / \mathrm{C}=\bigcup_{i=1}^{N} \Psi_{i}$. Then the contour $\mathrm{C}$ can be expressed as the union of the boundaries of the regions, denoted by $\mathrm{C}_{1}, \mathrm{C}_{2} \ldots \ldots \ldots \ldots \ldots \mathrm{C}_{\mathrm{N}}$, i.e. $\mathrm{C}=\bigcup_{i=1}^{N} C_{i}$. Therefore the above energy $f^{\mathrm{MS}}(u, \mathrm{C})$ can be equivalently written as

$$
\begin{aligned}
& f^{M S}\left(u_{1}, u_{2}, \ldots \ldots u_{N}, \Psi_{1}, \ldots . \Psi_{N}\right)= \\
& \sum_{i=1}^{N}\left(\int_{\Psi}\left(I-u_{i}\right)^{2} d x+\mu \int_{\Psi \backslash C}\left|\nabla u_{i}\right|^{2} d x+v\left|c_{i}\right|\right)
\end{aligned}
$$

Where $u_{\mathrm{i}}$ is the smooth function defined on the region $\Psi_{\mathrm{i}}$. The methods aiming to minimize this energy are called piecewise smooth models. This procedure is computationally expensive and more over the piecewise model is sensitive to the initialization of the contour C. In a variational level set formulation, Chan and Vese [2] simplified the Mumford-Shah functional as the following energy.

$$
\begin{aligned}
& f^{c v}\left(\Phi, c_{1}, c_{2}\right)=\int_{\Psi}\left|I(x)-c_{1}\right|^{2} H(\Phi(x)) d x \\
& +\int_{\Psi}\left|I(x)-c_{2}\right|^{2}(1-H(\Phi(x)) d x+ \\
& v \int_{\Psi}|\nabla H(\Phi(x))| d x
\end{aligned}
$$

where $\mathrm{H}$ is the Heaviside function, and $\Phi$ is a level set function [3]-[5]. The first two terms represent the data fitting terms, while the third term, with a weight $v>0$, regularizes the zero level contour. Image segmentation is therefore achieved by find the level set function $\Phi$ and the constants $c_{1}$ and $c_{2}$ that minimize the energy $f^{C V}$.

\section{Framework for Image Segmentation and Non-uniformity Correction}

(a) Image model and problem Statement:

In order to deal with intensity non-uniformities in image segmentation, segmentation is based on image model. Image model describes the composition of real world images in which intensity non-uniformities represented as a component of an image.

Here we consider the multiplicative model of intensity non-uniformity from the physics of imaging in a variety of modalities, an observed image I can be represented as

$$
\mathrm{I}=\mathrm{b}_{\mathrm{f}} \mathrm{T}+\mathrm{N}_{\mathrm{a}}
$$

where $\mathrm{T}$ is the real image, it measures the intrinsic physical property of objects being imaged which is assumed to be piecewise constant, and $b_{f}$ accounts for intensity non-uniformity, it is also called as bias field, $\mathrm{N}_{\mathrm{a}}$ is additive noise and it can be assumed to be zero mean Gaussian noise. Here we consider the image I as a function I: $\Psi \rightarrow \mathrm{R}$ defined on a continuous domain $\Psi$. The assumptions of real image and bias fields are explained in detailed as shown below:

[1] Consider a circular neighbourhood $O_{\mathrm{Y}}$, in this each pixel having different bias fields .The bias field $\mathrm{b}_{\mathrm{f}}$ is slowly varying and can be approximated by a constant in a neighbourhood.

[2] The real image $\mathrm{T}$ approximately divided into $\mathrm{N}$ distinct constant values $\mathrm{c}_{1} \ldots \ldots . \mathrm{c}_{\mathrm{N}}$ in disjoint regions $\Psi_{1} \ldots \ldots \ldots \Psi_{\mathrm{N}}$ respectively, where regions $\Psi_{1}$ $\Psi_{\mathrm{N}}$ forms a partition of the image domain

$$
\text { i.e. } \Psi=\bigcup_{i=1}^{N} \Psi_{i} \text { and } \quad \Psi_{\mathrm{i}} \cap \Psi_{\mathrm{J}}=\text { Null Set for } \mathrm{i} \neq j
$$


Based on the observed image and the above two assumptions, we proposed a method to estimate the regions $\underset{i=1}{N} \underset{i}{\Psi}$, the constants $\underset{i=1}{N} c_{i}$, and the bias field . The estimations of regions, constants and bias fields are denoted as N N

$\Psi_{i=1}, c_{i=1}$ and $\hat{\mathrm{b}}$. To avoid the spurious segmentation results caused by image noise, the bias field $\mathrm{b}_{\mathrm{f}}$ should be slowly varying and the regions should satisfy the certain regularity property. Based on the image model and the above two assumptions we will define a criterion. This criterion defined in terms of the regions $\Psi_{\mathrm{i}}$, constants $\mathrm{c}_{\mathrm{i}}$ and function $\mathrm{b}_{\mathrm{f}}$, as an energy in a variational framework, which is minimized for finding the optimal regions

$\stackrel{N}{\Lambda} \quad \stackrel{N}{\wedge}$

$\Psi_{i=1}$, constants $c_{i=1}$, and bias field $\hat{b}$. The result of framework and minimizing the energy in image segmentation and bias field estimation are simultaneously achieved.

(B) Local Intensity Clustering Property:

Region based image segmentation method typically depend on a specific region descriptor of the intensities of in each region to be segmented [6]. For example consider the seeded region based model, in this set of seeds as input along with the image. The seeds mark each of objects to be segmented. But this method is difficult to give such type of region descriptor for images with intensity non-uniformities. The overlap between the distributions of the intensities in the regions $\Psi_{1} \ldots \ldots \ldots \Psi_{\mathrm{N}}$ with the presence of intensity that's why it is impossible to segment these regions directly based on the pixel intensities. The property of local intensities is simple, which can be effectively exploited in the formulation of our method for image segmentation with simultaneous estimation of the bias field. Based on the observed image and assumptions we are able to derive a useful property of local intensities, which is referred to as a local intensity clustering property. Consider a circular neighbourhood with a radius $\rho$ centered at each point $\mathrm{y} \epsilon \Psi$ that is

$$
\mathrm{O}_{\mathrm{Y}} \stackrel{\Delta}{=}\{x:|x-y| \leq \rho\} \text {. }
$$

The partition region $\stackrel{N}{\Psi}_{i=1}^{N}$ of the entire domain $\Psi$ induces a partition of the neighbourhood of the $\mathrm{O}_{\mathrm{Y}}$, i.e, $\left\{\mathrm{O}_{Y} \stackrel{N}{\cap} \Psi_{i}\right\}$ forms a partition of $\mathrm{O}_{\mathrm{Y}}$. $i=1$ to $b_{\mathrm{f}}(\mathrm{y})$.

For a slowly varying bias field $b_{f}$, the values $b_{f}(x)$ for all $x$ in the circular neighbourhood $\mathrm{O}_{\mathrm{Y}}$ are close

$$
b_{\mathrm{f}}(\mathrm{x}) \approx \mathrm{b}_{\mathrm{f}}(\mathrm{y}) \quad \text { for } \mathrm{x} \in \mathrm{O}_{\mathrm{y}}
$$

Where $b_{f}(x)$ is the bias field with the function of $x, b_{f}(y)$ is the bias field with the function of $y$. Thus, the intensities $b_{\mathrm{f}}(\mathrm{x}) \mathrm{T}(\mathrm{x})$ in each sub-region $\mathrm{O}_{\mathrm{Y}} \cap \Psi_{\mathrm{i}}$ are close to the constant $\mathrm{b}_{\mathrm{f}}(\mathrm{y})$ that is

$$
\mathrm{b}_{\mathrm{f}}(\mathrm{x}) \mathrm{T}(\mathrm{x}) \approx \mathrm{b}_{\mathrm{f}}(\mathrm{y}) \mathrm{c}_{\mathrm{i}} \quad \text { for } \mathrm{x} \in \mathrm{O}_{\mathrm{y}} \cap \Psi_{\mathrm{i}}
$$

where $b_{f}(y) c_{i}$ is the constant, $b_{f}(y)$ is the bias field with the function of $y, b_{f}(x)$ is the bias field with the function of $\mathrm{x}$, and $\mathrm{T}(\mathrm{x})$ is the real image with the function of $\mathrm{x}$. substitute above equation in the observed image equation then we get

$$
\mathrm{I}(\mathrm{x}) \approx \mathrm{b}_{\mathrm{f}}(\mathrm{y}) \mathrm{c}_{\mathrm{i}}+\mathrm{N}_{\mathrm{a}}(\mathrm{x}) \quad \text { for } \quad \mathrm{x} \in \mathrm{O}_{\mathrm{y}} \cap \Psi_{\mathrm{i}}
$$

In the above equation $\mathrm{N}_{\mathrm{a}}(\mathrm{x})$ is the additive zero mean Gaussian noise. That is the intensities in the set ${\underset{Y}{I}}_{Y}^{i}\{\mathrm{I}(\mathrm{x})$ : $\left.\mathrm{x} \in \mathrm{O}_{\mathrm{y}} \cap \Psi_{\mathrm{i}}\right\}$ forms a cluster with center $\mathrm{m}_{\mathrm{i}} \approx \mathrm{b}_{\mathrm{f}}(\mathrm{y}) \mathrm{c}_{\mathrm{i}}$, which can be considered as samples drawn from a Gaussian distribution with mean $\mathrm{m}_{\mathrm{i}}$.

(C) Energy formulation:

By using the local intensity clustering property intensities in the neighbourhood $\mathrm{O}_{\mathrm{Y}}$ can be classified into $\mathrm{N}$ clusters, with centers $\mathrm{m}_{\mathrm{i}} \approx \mathrm{b}_{\mathrm{f}}(\mathrm{y}) \mathrm{c}_{\mathrm{i}}$. This property is applied to the standard $\mathrm{K}$ means clustering to classify these local intensities. For the intensities $\mathrm{I}(\mathrm{x})$ in the neighbourhood $\mathrm{O}_{\mathrm{Y}}$, the $\mathrm{K}$ means algorithm is an iterative process to minimize the clustering criterion. It can be written in a continuous form as

$$
F_{y}=\sum_{i=1}^{N} \int_{\mathrm{O}_{y}}\left|I(x)-m_{i}\right|^{2} u_{i}(x) d x
$$


Everyplace $m_{i}$ is the cluster center of the $i^{\text {th }}$ cluster, $u_{i}$ is the membership function of the region $\Psi_{i}$, i.e, $u_{i}(x)=1$ for $\mathrm{x} \in \Psi_{\mathrm{i}}, \mathrm{u}_{\mathrm{i}}(\mathrm{x})=0$ for $\mathrm{x}$ does not belongs to $\Psi_{\mathrm{i}}$ [3], [7], [8]. The above equation is in the form of energy equation $\mathrm{F}=\int_{\mathrm{X}} \mathrm{dx}$. Then the above equation can be rewritten as

$$
F_{y}=\sum_{i=1}^{N} \int_{\Psi_{i} \cap o_{y}}\left|I(x)-m_{i}\right|^{2} d x
$$

In view of the clustering criterion in above equation and the approximation of the cluster center by $\mathrm{m}_{\mathrm{i}} \approx \mathrm{b}_{\mathrm{f}}(\mathrm{y}) \mathrm{c}_{\mathrm{i}}$. Clustering criterion for classifying the intensities in $\mathrm{O}_{\mathrm{Y}}$ as

$$
R_{y}=\sum_{i=1}^{N} \int_{\Psi_{i} \cap o_{y}} k(y-x)\left|I(x)-b_{f}(y) c_{i}\right|^{2} d x
$$

Where $k(y-x)$ is non-negative window function, also called as Kernel function [9]. $\mathrm{K}(\mathrm{y}-\mathrm{x})=0$ for $\mathrm{x}$ does not belongs to $\mathrm{O}_{\mathrm{Y}}$. With the window function, the clustering criterion function $\mathrm{R}_{\mathrm{y}}$ can be rewritten as

$$
R_{y}=\sum_{i=1}^{N} \int_{\Psi_{i}} k(y-x)\left|I(x)-b_{f}(y) c_{i}\right|^{2} d x
$$

This local clustering criterion function is a basic element in the formulation of our method. The local clustering criterion function $\mathrm{R}_{\mathrm{y}}$ evaluates the classification of the intensities in the neighbourhood $\mathrm{O}_{\mathrm{Y}}$ given by the partition

$\left\{\mathrm{O}_{Y} \stackrel{N}{\cap} \Psi_{i=1}\right\}$ of $\mathrm{O}_{\mathrm{Y}}$. The smaller value of the $\mathrm{R}_{\mathrm{y}}$ is the better classification.

We define the optimal partition $\underset{i=1}{\stackrel{N}{\Psi}}$ of the entire domain $\Psi$. Such that the local clustering criterion function $\mathrm{R}_{\mathrm{y}}$ is minimized for all $\mathrm{y}$ in $\Psi$. Then we need to jointly minimize $\mathrm{R}_{\mathrm{y}}$ for all $\mathrm{y}$ in the $\Psi$. This can be achieved by minimizing the integral of $\mathrm{R}_{\mathrm{y}}$ with respect to y over the image domain $\Psi$.We define an energy

$$
\begin{gathered}
\mathrm{R}=\int \mathrm{R}_{\mathrm{y}} \mathrm{dy} \\
R=\int\left[\sum_{i=1}^{N} \int_{\psi_{i}} k(y-x)\left|I(x)-b_{f}(y) c_{i}\right|^{2} d x\right] d y
\end{gathered}
$$

We omit the domain $\Psi$ in the subscript of the integral symbol if the integration is over the entire domain $\Psi$. By minimizing the energy with respect to the regions $\Psi_{1} \ldots \ldots . \Psi_{\mathrm{N}}$, constants $\mathrm{c}_{1} \ldots \ldots . \mathrm{c}_{\mathrm{N}}$ and the bias field $\mathrm{b}_{\mathrm{f}}$, we can estimate the bias field and image segmentation. The choice of kernel function $\mathrm{k}$ is flexible. For example it can be a truncated uniform function, defined as $\mathrm{K}(\mathrm{u})=\mathrm{a}$ for $|\mathrm{u}| \leq \rho$ and $\mathrm{k}(\mathrm{u})=0$ for $|\mathrm{u}|>\rho$. If a is positive constant then $\int \mathrm{k}(\mathrm{u})=1$. Then kernel function $\mathrm{k}$ is chosen as a truncated Gaussian function defined by

$$
k(u)= \begin{cases}(1 / e) \ell^{-|u|^{2}} / 2 \sigma^{2}, f o r|u| \leq \rho \\ 0, & \text { otherwise }\end{cases}
$$

where $\mathrm{e}$ is the normalized constant, $\sigma$ is the standard deviation of the Gaussian noise, $\rho$ is the radius of the neighbourhood $\mathrm{O}_{\mathrm{Y}}$

According to the degree of the intensity non-uniformity the neighbourhood $\mathrm{O}_{\mathrm{Y}}$ and the radius $\rho$ should be selected. For more localized intensity non-uniformity the bias field $\mathrm{b}$ varies faster and the approximation of the equation $\mathrm{b}_{\mathrm{f}}(\mathrm{x}) \approx \mathrm{b}_{\mathrm{f}}(\mathrm{y})$ for $\mathrm{x} \in \mathrm{O}_{\mathrm{y}}$. The above equations are valid only in the smaller neighbourhood. A smaller $\rho$ should be used as the radius of the neighbourhood $\mathrm{O}_{\mathrm{Y}}$.

\section{Level Set Formulation and Energy Minimization}

In level set formulation, level set function takes only positive and negative signs and this represents a part in the image $\Psi$. From the expression of $\epsilon$ we cannot derive solution to the energy minimization problem. And by using well- established variational methods, the energy minimization can be solved in level set formulation. Consider $\phi: \Psi \rightarrow \mathrm{R}$ represents a level set function, and then it defines two disjoint regions

$$
\Psi_{1}=\{\mathrm{x}: \phi(\mathrm{x})>0\}
$$$$
\Psi_{2}=\{\mathrm{x}: \phi(\mathrm{x})<0\}
$$

There are two types of level set formulation. They are

(1) Two phase level set Formulation ( $\mathrm{N}=2)$

(2) Multiphase level set formulation( $\mathrm{N}>2)$

(1)Two phase level set formulation:

In this case the image domain is partitioned into two disjoint regions $\Psi_{1}$ and $\Psi_{2}$ and a level set function is $\phi$ is used to represent the disjoint regions. Here the two disjoint regions $\Psi_{1}$ and $\Psi_{2}$ are represented with their 
membership functions defined by $\mathrm{M}_{1}(\phi)=\mathrm{H}(\phi)$ and $\mathrm{M}_{2}(\phi)=1-\mathrm{h}(\phi)$, where $\mathrm{H}$ is the Heaviside function [10], [11]. We can define energy as

$$
\mathrm{R}=\int\left[\sum_{i=1}^{N} \int_{\Psi_{i}} k(y-x)\left|I(x)-b_{f}(y) c_{i}\right|^{2} d x\right] \mathrm{dy}
$$

Thus for $\mathrm{N}=2$ case the above energy equation can be expressed in terms of the level set formulation

$$
R=\int\left(\sum_{i=1}^{N} \int k(y-x)\left|I(x)-b_{f}(y) c_{i}\right|^{2} M_{i}(\Phi(x)) d x\right) d y
$$

By exchanging the order of integration we have

$$
R=\int\left(\sum_{i=1}^{N} \int k(y-x)\left|I(x)-b_{f}(y) c_{i}\right|^{2} d y\right) M_{i}(\Phi(x)) d x
$$

The variables of the energy $R$ can be written as $R\left(\phi, C, b_{f}\right)$ where $C$ represents the constants $c_{1} \ldots \ldots c_{N}$ with a vector $\mathrm{c}, \phi$ represents the level set function and $\mathrm{b}_{\mathrm{f}}$ represents the bias field [12]. The energy $\mathrm{R}\left(\phi, \mathrm{C}, \mathrm{b}_{\mathrm{f}}\right)$ can be rewrite as

$$
R\left(\Phi, C, b_{f}\right)=\int\left(\sum_{i=1}^{N} e_{i}(x) M_{i}(\Phi(x)) d x\right.
$$

where $e_{i}$ is given by

$$
e_{i}(x)=\int k(y-x)\left|I(x)-b_{f}(y) c_{i}\right|^{2} d y
$$

The equivalent expression for the function $\mathrm{e}_{\mathrm{i}}$ can be defined as

$$
e_{i}(x)=I^{2} 1_{k}-2 c_{i} I\left(b_{f} * k\right)+c_{i}^{2}\left(b_{f}{ }^{2} * k\right)
$$

Where $*$ is the convolution operator and $1_{\mathrm{k}}=\int \mathrm{k}(\mathrm{y}-\mathrm{x})$ which is equal to constant 1 , except near the boundary of the image $\Psi$ [13]. The variational level set formulation is defined by

$$
F\left(\phi, c, b_{f}\right)=R\left(\phi, c, b_{f}\right)+v L(\phi)+\mu R_{p}(\phi)
$$

Where $\mathrm{L}(\phi)$ and $\mathrm{R}_{\mathrm{p}}(\phi)$ can be defined as the regularization terms

$$
\mathbf{L}(\phi)=\int|\nabla H(\phi)| d x
$$

The $\mathrm{L}(\phi)$ represents the arc length of the contour and then it smooth the contour

$$
R_{p}(\phi)=\int p(|\nabla \phi|) d x
$$

The $\mathrm{R}_{\mathrm{p}}(\phi)$ is also called as distance regularization term and in different general variational level set formulation is called as distance regularized level set evolution [14]. The level set function $\phi$ and the bias field estimation gives the result of image segmentation and this can be obtained by minimizing the energy. The energy can be minimized in iteration process, and in each iteration the minimizing can be done by doing partial differentiation of $\mathrm{F}\left(\phi, \mathrm{C}, \mathrm{b}_{\mathrm{f}}\right)$ with respect to $\phi$, c, and $\mathrm{b}_{\mathrm{f}}$.

(1) Energy Minimization with respect to $\phi$ :

By making $\mathrm{C}$ and $\mathrm{b}$ as fixed, the minimization of $\mathrm{F}\left(\phi, \mathrm{C}, \mathrm{b}_{\mathrm{f}}\right)$ with respect to $\phi$ can be achieved by using standard gradient descent method.

$$
\frac{\partial \phi}{\partial t}=-\frac{\partial f}{\partial \phi} \quad \text { where } \frac{\partial f}{\partial \phi} \text { is the gateaux derivative. }
$$

By minimizing the energy $\mathrm{R}\left(\phi, \mathrm{C}, \mathrm{b}_{\mathrm{f}}\right)$ with respect to $\mathrm{C}$ and $\mathrm{b}_{\mathrm{f}}$, the constants $\mathrm{c}_{1}$ and $\mathrm{c}_{2}$ in $\mathrm{C}$ and the bias field $b_{f}$ are updated during the level set formulation.

(2) Energy minimization with respect to C:

By making $\phi$ and $\mathrm{b}$ as fixed, the optimal $\mathrm{C}$ that minimizes the energy $\mathrm{R}\left(\phi, \mathrm{C}, \mathrm{b}_{\mathrm{f}}\right)$ denoted by $\hat{\mathrm{C}}=\left(\hat{\mathrm{C}}_{\mathrm{i}} \ldots \ldots \hat{\mathrm{C}}_{\mathrm{n}}\right)$ by, is given by

$$
\hat{\mathrm{C}}_{\mathrm{i}}=\frac{\int\left(b_{f} * k\right) I u_{i} d y}{\int\left(b_{f}{ }^{2} * k\right) u_{i} d y}, i=1,2, \ldots . . N \quad \mathrm{u}_{\mathrm{i}}(\mathrm{y})=\mathrm{M}_{\mathrm{i}}(\varnothing(\mathrm{y})) .
$$

(3) Energy minimization with respect to $b_{f}$ :

By making $\phi$ and $\mathrm{C}$ as fixed, the optimal $\mathrm{b}$ that minimizes the energy $\mathrm{R}\left(\phi, \mathrm{C}, \mathrm{b}_{\mathrm{f}}\right)$ denoted by $\hat{\mathrm{b}}_{\mathrm{f}}$

$$
\hat{\mathrm{b}}_{\mathrm{f}}=\frac{\left(I T^{(1)}\right)^{*} K}{T^{(2)} * K} \quad \text { where } \quad T^{(1)}=\sum_{i=1}^{N} c_{i} u_{i} \text { and } T^{(2)}=\sum_{i=1}^{N} c_{i}{ }^{2} u_{i}
$$


The slowly varying property of the derived optimal estimator $\hat{b}_{\mathrm{f}}$ is convoluted with kernel function $\mathrm{k}$.

\section{Multi-Phase Level set Formulation:}

In this case we use two or more level set functions $\phi_{1}$

$$
\begin{aligned}
& \mathrm{M}_{1}\left(\emptyset_{1}, \emptyset_{2}\right)=\mathrm{H}\left(\emptyset_{1}, \emptyset_{2}\right), \\
& \mathrm{M}_{2}\left(\emptyset_{1}, \emptyset_{2}\right)=\mathrm{H}\left(\emptyset_{1}\right)\left(1-\mathrm{H}\left(\emptyset_{2}\right)\right), \\
& \mathrm{M}_{3}\left(\emptyset_{1}, \emptyset_{2}\right)=1-\mathrm{H}\left(\emptyset_{1}\right)
\end{aligned}
$$

.$\phi_{\mathrm{k}}$ to define $\mathrm{N}$ membership functions [7].

And the above functions gives the three phase level set formulations. The energy in the first equation can be converted to a multiphase level set formulation is expressed as

$$
R\left(\Phi, C, b_{f}\right)=\int\left(\sum_{i=1}^{N} e_{i}(x) M_{i}(\Phi(x)) d x\right.
$$

The energy functional $\mathrm{F}$ in multiphase level set formulation is given by

$$
\mathrm{F}\left(\phi, \mathrm{C}, \mathrm{b}_{\mathrm{f}}\right) \stackrel{\Delta}{=} \mathrm{R}\left(\phi, \mathrm{C}, \mathrm{b}_{\mathrm{f}}\right)+\mathrm{R}_{p}(\phi)
$$

The minimization of the energy $\varepsilon(\phi, \mathrm{C}, \mathrm{b})$ can be achieved by the same procedure as in the two-phase case [15], [16].

\section{Simulation RESULTS AND DISCUSSIONS}

We first demonstrate our method in the two-phase case (i.e. $\mathrm{N}=2$ ). Unless otherwise specified, the parameter $\sigma$ is set to 4 for the experiments in this section. All the other parameters are set to the default values. Fig. 1 shows the results for a MRI image. The curve evolution processes are depicted by showing the initial contours (in the left column), bias field (in the middle column).
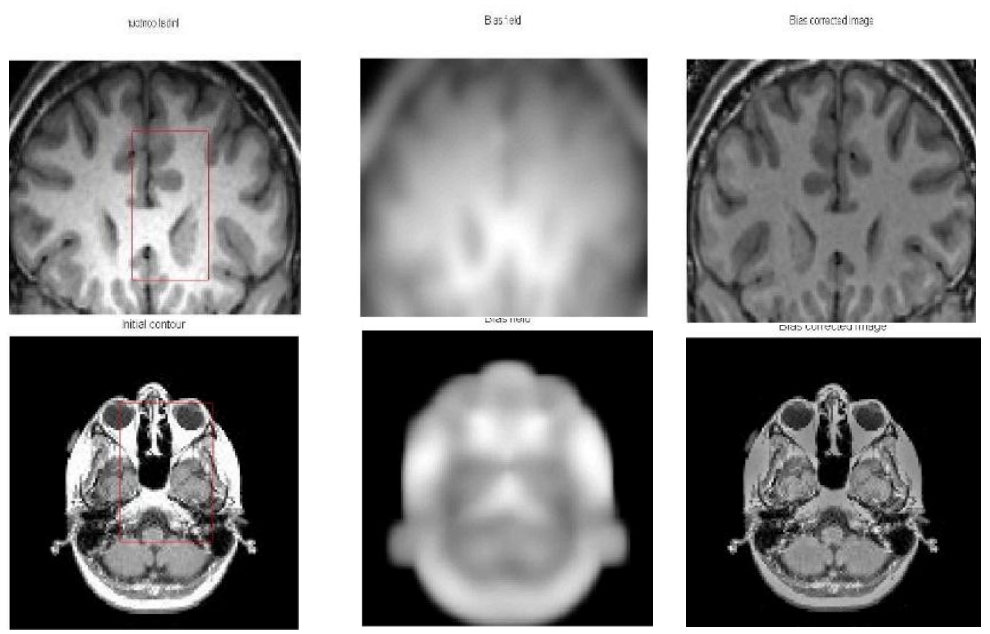

Fig. 1: Segmentation for MR images.

Intensity non-uniformities can be clearly seen in these two images. Our method is able to provide a desirable segmentation result for such images. The estimated bias field by our method can be used for intensity nonuniformity correction (or bias correction). Given the estimated bias field, the bias corrected image is computed as the quotient $\mathrm{I} / \hat{b}_{\mathrm{f}}$. To demonstrate the effectiveness of our method in simultaneous segmentation and bias field estimation, we applied it to medical images with intensity non-uniformities. These images exhibit clear intensity non-uniformities. The initial contour is plotted on the original image in Column 1 of Fig.2. The corresponding results of segmentation, bias field estimation, and bias correction are shown in Columns 2, 3 and 4, respectively. These results demonstrate desirable performance of our method in segmentation and bias correction. We first display the results for MR images in the first column of Fig. 2. These images exhibit obvious intensity nonuniformities. The segmentation results, computed bias fields, bias corrected images, are shown in the second, third, and fourth column respectively. It can be seen that the intensities within each tissue become quite homogeneous in the bias corrected images. The improvement of the image quality in terms of intensity homogeneity can be also demonstrated by comparing the histograms of the original images and the bias corrected images. 

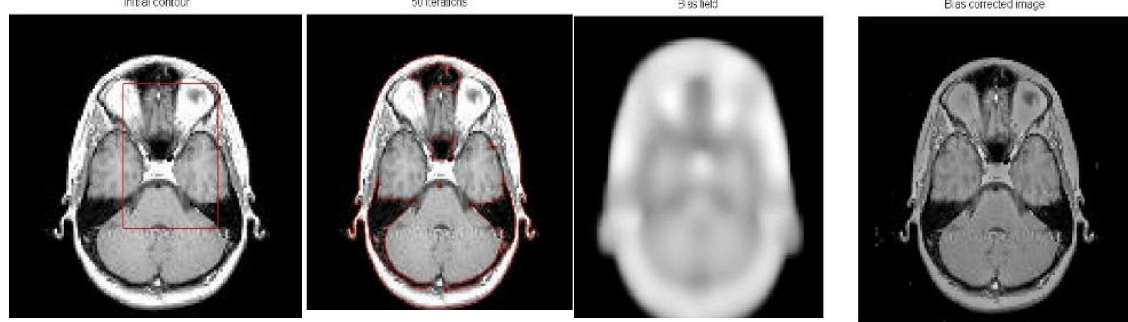

Fig. 2: Simulation Results with MR images as input
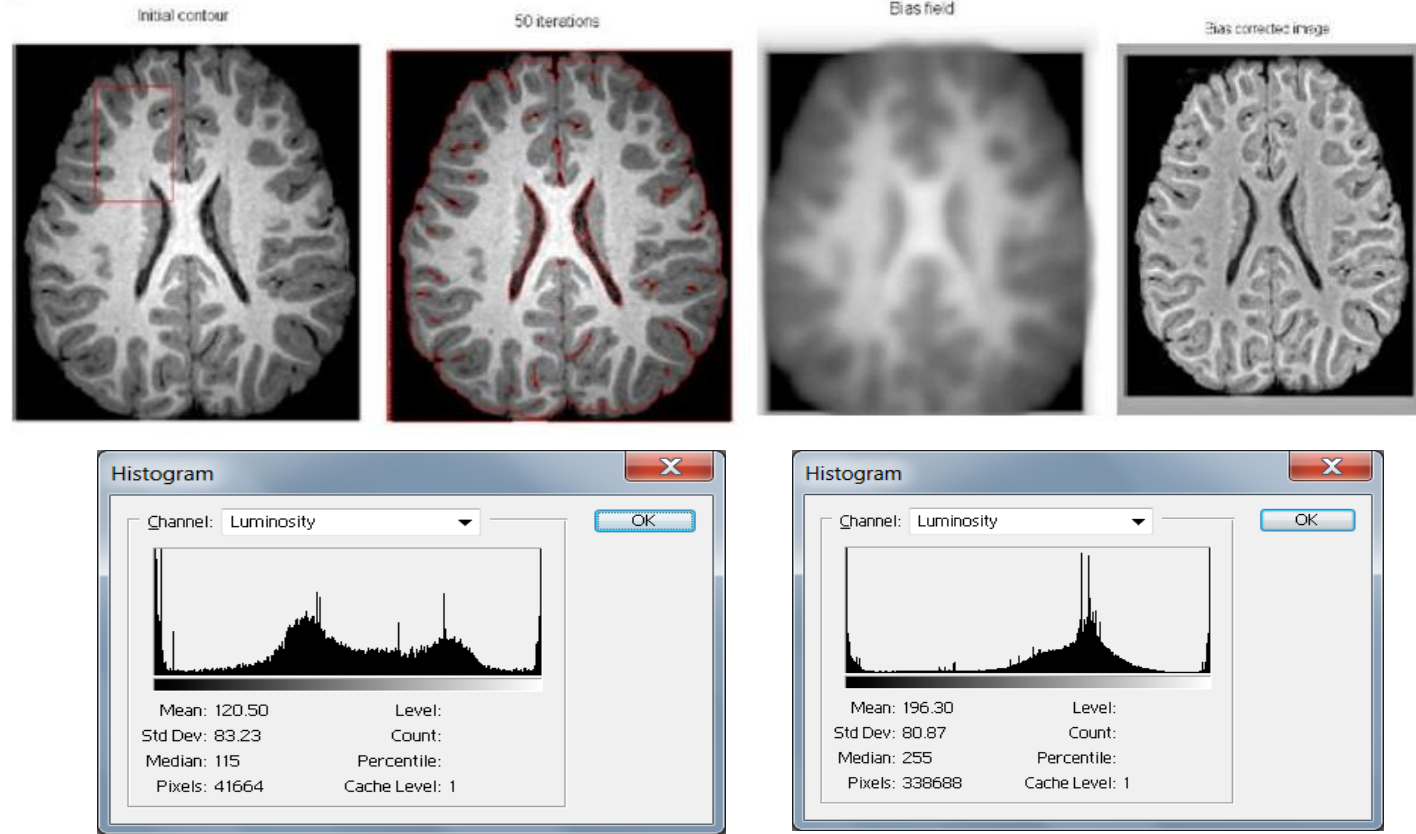

Fig. 3: Simulation Results with another MR Image as input along with Histograms

The histograms of the original images (left) and the bias corrected images (right) are plotted in the fifth column. The results of the real world images are as follows. In the fig. 4 , it consists of the different modules in the real world images such as 50 iterations in column-1,bias field in column-2, Bias corrected in column-3, and the original image in column- 4 , and the histogram is generated between the original image and the bias corrected image as shown in the fig 3 .
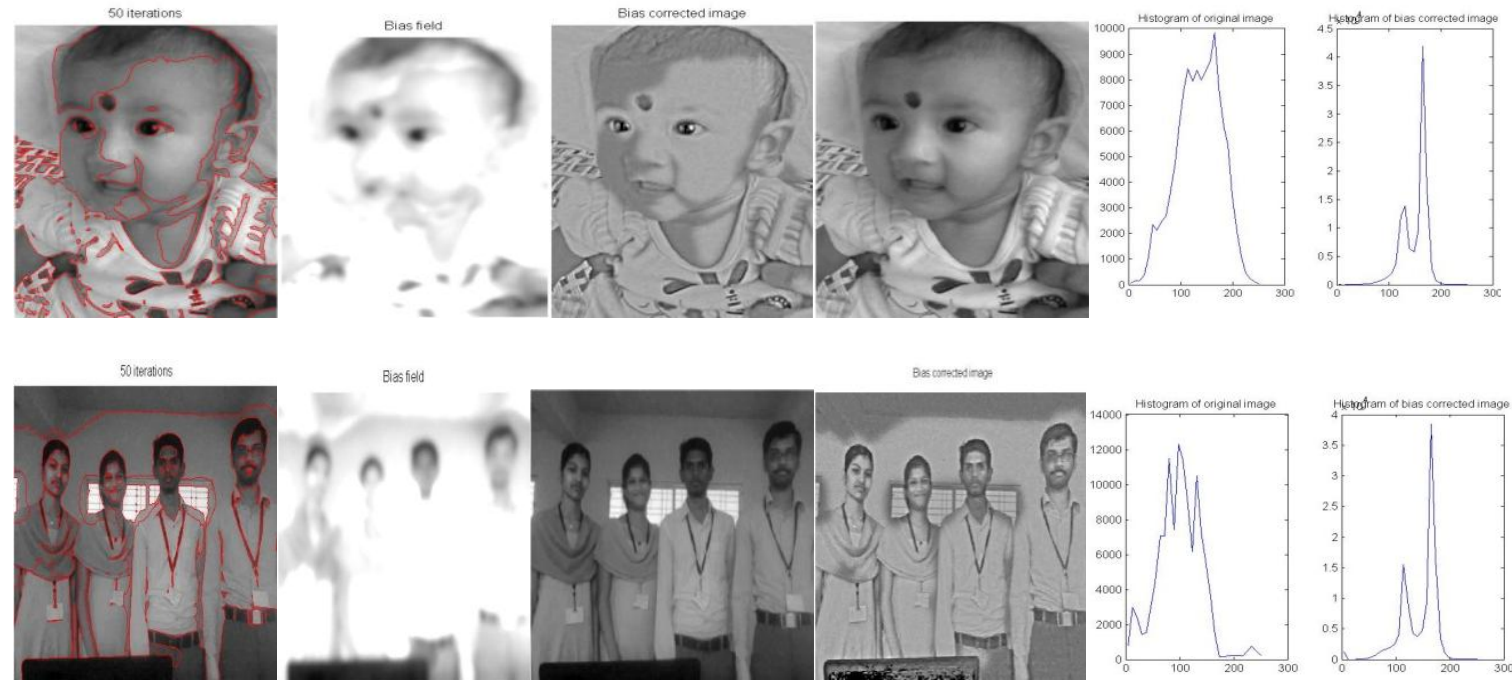

Fig. 4: Simulation Results with another MR Image as input along with Histograms 
The results of novel region based method when applied on the real world images in multiphase are shown below:

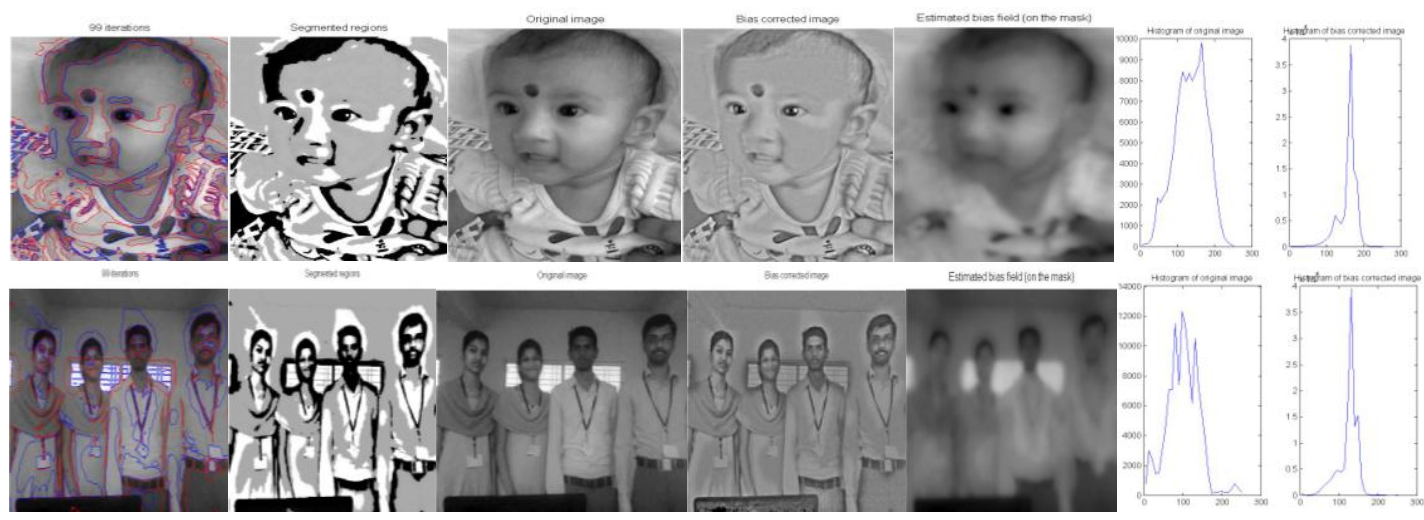

Fig 5: Simulation Results of Multiphase algorithm with Real world Images as input along with Histograms.

The present work can be extended by considering a specialized database or set of images of different categories. Also by considering rectangular neighbourhood rather than circular neighborhood the overlapping of intensity regions can be avoided well.

\section{CONCLUSIONS}

We have presented a variation level set framework for segmentation and bias correction of images with intensity non-uniformities. Based on a generally accepted model of images with intensity non-uniformities and a derived local intensity clustering property, we define energy of the level set functions that represent a partition of the image domain and a bias field that accounts for the intensity non-uniformity. Segmentation and bias field estimation are therefore jointly performed by minimizing the proposed energy functional. The slowly varying property of the bias field derived from the proposed energy is naturally ensured by the data terminal our variation framework, without the need to execute a clear smoothing term on the bias field. Our method is much more robust to initialization than the piecewise smooth model. Experimental results have demonstrated superior performance of our method in terms of accuracy, efficiency, and robustness. As an application, our method has been applied to MR image segmentation and bias correction with promising results.

\section{Acknowledgements}

At the outset we express our gratitude to Dr. P Gopal Reddy sir, Director, PDCE, Sullurpet, Dr. A Siva Sankar sir, Principal, PDCE, Sullurpet, and the faculty members Y Ravi Sankaraiah sir, MS Abdullah sir, B Chandra Sekhar sir, V Kiran Kumar sir, S Sri Harshith sir, M Chiranjeevi sir, M Balaji Kumar sir, P Srinivasulu sir, M Gangadhar sir, E Sasikala madam, K Bharathi madam, G Sumalatha madam, B Mahendra sir and D Baba Prasad sir who have guided us in completing this work with their esteemed cooperation and valuable guidance, and who has given our work a shape and success.

\section{Journal Papers:}

\section{REFERENCES}

[1] Chunming Li, Rui Huang, Zhaohua Ding, J. Chris Gatenby, Dimitris N. Metaxas, and John C. Gore, “A Level Set Method for Image Segmentation in the Presence of Intensity Inhomogeneities With Application to MRI", IEEE Trans. Image. Process., vol. 20, no. 7, July 2011.

[2] T. Chan and L. Vese, "Active contours without edges," IEEE Trans. Image. Process., vol. 10, no. 2, pp. 266-277, Feb. 2001.

[3] R. Ronfard, "Region-based strategies for active contour models," Int. J. Comput. Vis., vol. 13, no. 2, pp. 229-251, Oct. 1994.

[4] C. Samson, L. Blanc-Feraud, G. Aubert, and J. Zerubia, "A variational model for image classification and restoration," IEEE Trans. PatternAnal. Mach. Intell., vol. 22, no. 5, pp. 460-472, May 2000.

[5] S. Osher and J. Sethian, "Fronts propagating with curvature-dependent speed: Algorithms based on Hamilton-Jacobi formulations," J. Comp.Phys., vol. 79, no. 1, pp. 12-49, Nov. 1988.

[6] V. Caselles, F. Catte, T. Coll, and F. Dibos, "A geometric model for active contours in image processing," Numer. Math., vol. 66, no. 1, pp. 1-31, Dec. 1993.

[7] D. Cremers, “A multiphase level set framework for variational motion segmentation," in Proc. Scale Space Meth. Comput. Vis., Isle of Skye, U.K., Jun. 2003, pp. 599-614.

[8] C. Li, R. Huang, Z. Ding, C. Gatenby, D. Metaxas, and J. Gore, "A variational level set approach to segmentation and bias correction of medical images with intensity Inhomogeneity," in Proc. Med. Image Comput. Aided Intervention, 2008, vol. LNCS 5242, pp.10831091, Part II.

[9] C. Li, C. Kao, J. C. Gore, and Z. Ding, "Minimization of region-scalable fitting energy for image segmentation," IEEE Trans. ImageProcess, vol. 17, no. 10, pp. 1940-1949, Oct. 2008. 
[10] C. Li, C. Xu, C. Gui, and M. D. Fox, "Distance regularized level set evolution and its application to image segmentation," IEEE Trans. ImageProcess., vol. 19, no. 12, pp. 3243-3254, Dec. 2010.

[11] R. Malladi, J. A. Sethian, and B. C.Vemuri, "Shape modeling with front propagation: A level set approach," IEEE Trans. Pattern Anal. Mach.Intell., vol. 17, no. 2, pp. 158-175, Feb. 1995.

[12] N. Paragios and R. Deriche, "Geodesic active contours and level sets for detection and tracking of moving objects," IEEE Trans. Pattern Anal.Mach. Intell., vol. 22, no. 3, pp. 266-280, Mar. 2000.

[13] V. Caselles, R. Kimmel, and G. Sapiro, "Geodesic active contours," Comput. Vis., vol. 22, no. 1, pp. 61-79, Feb. 1997.

[14] S. Kichenassamy, A. Kumar, P. Olver, A. Tannenbaum, and A. Yezzi, "Gradient flows and geometric active contour models," in Proc. 5th Int.Conf. Comput. Vis., 1995, pp. 810-815.

[15] N. Paragios and R. Deriche, "Geodesic active regions and level set methods for supervised texture segmentation," Int. J. Comput. Vis., vol. 46, no. 3, pp. 223-247, Feb. 2002.

[16] D. Mumford and J. Shah, "Optimal approximations by piecewise smooth functions and associated variational problems," Commun.Pure Appl. Math., vol. 42, no. 5, pp. 577-685, 1989.

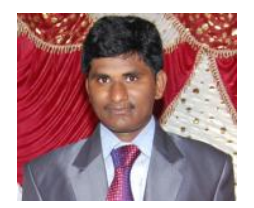

Jaya Krishna Sunkara received B.Tech in Electronics and Communication Engineering from GKCE, Jawaharlal Nehru Technological University in 2006 and M.E in Information Technology from Bangalore University in 2009. Since 2009, he is in PDCE, Sullurpet, as an Asst. Prof., in ECE Dept. He stood University first in ME and also qualified in GATE for 6 times, thrice in EC and thrice in CS. His research areas include Image Processing and Programming Methodologies.

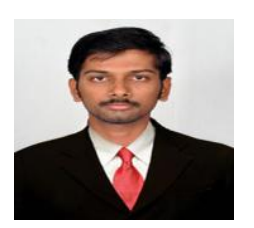

Sundeep Eswarawaka, a student of B.Tech in Electronics and Communication Engineering, in PDCE, Jawaharlal Nehru Technological University, currently working on Image Processing. His research interests are Image Segmentation, DCT Variations, Coding Techniques and MATLAB Implementations.

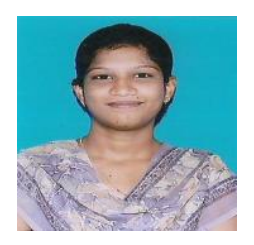

Kiranmai Darisi, a student of B.Tech in Electronics and Communication Engineering, in PDCE, Jawaharlal Nehru Technological University, currently working on Image Processing. Her research interests are Implementations of different phenomenon in MATLAB and Medical Image analysis.

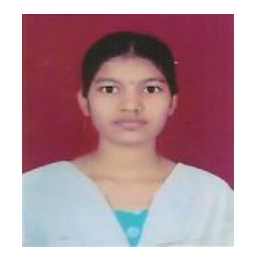

Santhi Dara, a student of B.Tech in Electronics and Communication Engineering, in PDCE, Jawaharlal Nehru Technological University, currently working on Image Processing. Her research interests are Video Compression, DWT Variations, Coding Techniques and MATLAB Implementations.

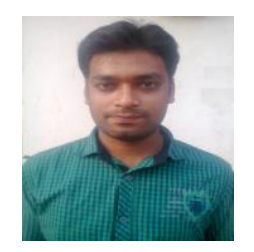

Pushpa Kumar Dasari, a student of B.Tech in Electronics and Communication Engineering, in PDCE, Jawaharlal Nehru Technological University, currently working on Image Processing. His research interests are Image Segmentation, and MATLAB Implementations.

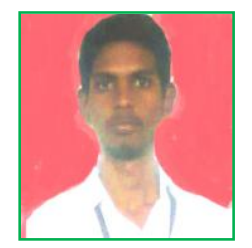

Prudhviraj Dara, a student of B.Tech in Electronics and Communication Engineering, in PDCE, Jawaharlal Nehru Technological University, currently working on Image Processing. His research interests are Image Segmentation, and DCT Variations. 